\title{
EFEITOS DO FRACIONAMENTO DA ADUBAÇÃO MINERAL, DA ADUBAÇÃO ORGÂNICA E DA CALAGEM NA PRODUTIVIDADE E INCIDÊNCIA DE VASSOURA-DE-BRUXA EM CACAUEIROS DA AMAZÔNIA(1)
}

\author{
F. I. O. MORAIS ${ }^{(2)}$
}

RESUMO

\begin{abstract}
A resposta do cacaueiro à aplicação de micronutrientes, matéria orgânica, calcário e fracionamento da adubação foi determinada em dois experimentos, instalados em solos das unidades Terra R oxa estruturada eutrófica (TR) e L atossolo Amarelo (LA), no Estado do Pará, no período de 1988 a 1993. As lavouras de cacau do híbrido Sca 6 x Be 10 foram implantadas pelo sistema de derrubada total e queima da floresta primária, utilizando-se sombreamento provisório de bananeira (Musa spp.) e permanente de Erythrina poeppigiana ou Gmelina arborea. Os tratamentos foram distribuídos em blocos casualizados, com três repetições, sendo a parcela constituída de 20 plantas úteis. Os resultados de produção de cacau demonstraram que não houve interação si gni ficativa entre tratamentos e solos ou ano de condução do experimento. $O$ fracionamento da adubação NPK em três aplicações/ano e a adubação NPK $+\mathrm{Zn}$ foram os melhores tratamentos, provocando incrementos $(P<0,05)$ na produtividade do cacaueiro da ordem de $27,5 \%$ e $10,9 \%$, respectivamente. A menor incidência $(P<0,05)$ de frutos atacados pela enfermidade vassoura-de-bruxa, causada pelo fungo Crinipellis perniciosa, foi observada nos tratamentos em que se aplicaram uma mistura de micronutrientes (B, Cu, Zn, Fe e Mo) ou esterco de gado na dosagem de $5 \mathrm{t} \mathrm{ha}^{-1}$; as porcentagens de frutos doentes dos demais tratamentos não diferiram da adubação NPK (testemunha). Para o aumento da produtividade, a estratégia de adubação mais eficiente foi o fracionamento da adubação NPK ( $60 \mathrm{~kg} \mathrm{ha}^{-1}$ de N, $\mathrm{P}_{2} \mathrm{O}_{5}$ e $\left.\mathrm{K}_{2} \mathrm{O}\right)$ em três aplicações ao ano. $O$ efeito de micronutrientes e do esterco de gado na infecção da vassoura-debruxa merece ser investigado com mais atenção.
\end{abstract}

Termos de indexação: micronutrientes, NPK, esterco de gado, Terra Roxa estruturada, Latossolo Amarelo.

\footnotetext{
(1) Recebido para publicação em outubro de 1994 e aprovado em dezembro de 1997.

(2) Pesquisador Titular da CEPLAC, aposentado, Professor Visitante da Faculdade de Ciências Agrárias do Pará. Rua Tiradentes, 590/ 701. CEP 66053-330 Belém (PA).
} 


\title{
SUMMARY: EFFECTS OF FERTILIZATION, MANURING AND LIMING ON YIELD AND INCIDENCE OF WITCHES' BROOM IN CACAO TREES OF THE AMAZON BASIN OF BRAZIL
}

\begin{abstract}
The effects of applying micronutrients, cattle manure, lime and splitting application of NPK fertilizers on the yield and the incidence of witches' broom disease of cacao caused by Crinipellis perniciosa were determined in two experiments carried out on structured Terra Roxa soil (TR) and Yellow Latosol (LA), in Pará State, from 1988 to 1993, after the slash and burn of theforest. Thehybrid (Sca 6 x Be 10) cacao crop was introduced utilizing banana (Musa spp.), as temporary shading, and a leguminous tre(Erythrina poeppigiana) or Gmel ina arborea, as permanenteshading, Theexperimental design was a randomized block with threereplications of 20 cacao trees per pl ot in each soil. Cacao yiedd data showed that there was no interaction between treatments and soils or cropping year. The highest yiel ds were obtained by splitting NPK fertilizers three times a year or by applying NPK plus zinc, which increased $(P<0,05)$ production by $27,5 \%$ and $10,9 \%$, respectively. Lowest witches' broom pod infection $(P<0,05)$ occurred when soil was treated with a micronutrient mix (Bo, Cu, Zn, Feand Mo) or with cattle manure at the rate of $5 \mathrm{t} \mathrm{ha}^{-1}$. The most promi sing fertilizer strategy to increase yiel d of cocoa was splitting the rate of a NPK fertilizer (60 kg ha-1 of N, $\mathrm{P}_{2} \mathrm{O}_{5}$ and $\left.\mathrm{K}_{2} \mathrm{O}\right)$ threetimes a year. The importance of micronutrients, cattle manure and liming on pod infection by witches' broom disease deserves more attention in future research.
\end{abstract}

Index terms: micronutrient, NPK, cattle manure, Yellow Latosol , Terra Roxa.

\section{INTRODUÇÃO}

A cacauicultura da Amazônia foi implantada em áreas de acentuada diversidade pedológica, com predominância de latossolos e podzólicos distróficos, e, em menor escala, de solos eutróficos, especialmente Terra Roxa (Vieira et al., 1967; Falesi, 1972; N eves \& Barbosa, 1982).

Experimentos real izados em casa de vegetação por Campos (1982) demonstraram que o $P$ foi o nutriente mais limitante para o desenvol vimento de plântulas de cacau nesses solos; oK, o Mg e o Zn foram também deficientes nos solos de baixa fertilidade natural. $\mathrm{Na}$ fase de crescimento do cacaueiro cultivado em Latossolo Amarelo, em condições de campo, o P foi o nutriente que provocou o maior incremento no diâmetro do caule, observando-se a ocorrência de interações significativas $\mathrm{N}$ × P e P x K. A estratégia mais indicada deadubação foi a incorporação de adubo orgânico na cova de plantio ea aplicação subsequente de uma mistura fertilizante NPK a lanço, anual mente, ou o fracionamento da adubação NPK em três aplicações ao ano. Não houve respostas significativas para macronutrientes, micronutrientes, calagem ou adubação orgânica na Terra Roxa (Morais, 1988).

A vassoura-de-bruxa, causada pelo fungo Crinipel lis pernici osa, éa mais importante doença do cacaueir na Amazônia. Os basidiosporos produzidos pelo fungo possuem a capacidade de penetrar em qualquer tecido de crescimento ativo do cacaueiro, causando a hipertrofia de brotos vegetativos, destruição de al mofadas florais e perdas de até $90 \%$ na produção de cacau (Bastos \& Evans, 1979;
Lawrence et al., 1991). Apesar de sua importância econômica, nenhum trabalho foi realizado sobre a interação adubação x infecção de vassoura-de-bruxa do cacaueiro.

Este artigo apresenta e discute os dados obtidos sobre o efeito de mi cronutrientes, adubação orgânica, calagem e fracionamento da adubação mineral na produtividade e na infecção da vassoura-de-bruxa em cacaueiros da Amazônia brasileira.

\section{MATERIAL E MÉTODOS}

O experimento foi desenvolvido, entre 1988 e 1993, em duas plantações de cacau estabel eci das no ano de 1982, em sol os das unidades Terra Roxa estruturada eutrófica (TR) e Latossolo Amarelo (LA), no Pará. As lavouras de cacau do híbrido Sca 6 x Be 10 foram implantadas pelo sistema de derrubada total equeima da vegetação, no espaçamento de $3 \times 3 \mathrm{~m}$, sob sombreamento provisório de bananeira (Musa spp.) e permanente de eritrina (Erythrina poeppigiana), no soloTR, ou de Gmelina arborea, no LA, obedecendo à densidade de plantio recomendada por Garcia et al . (1985). As propriedades físicas e químicas dos solos estão contidas no quadro 1, tendo sido descritas por Morais (1988).

O delineamento experimental foi em blocos casualizados, com três repetições, em um esquema fatorial NPK 23, com tratamentos adicionais defósforo, zinco, calagem, adubação orgânica, micronutrientes (Cu, Zn, Mo, Fe e B) e fracionamento de uma mistura 
Quadro 1. Propriedades químicas e físicas de amostras dos solos

\begin{tabular}{|c|c|c|c|c|c|c|c|c|c|c|c|c|}
\hline Solo ${ }^{(1)}$ & Profundidade & Areia & Silte & Argila & C & pH & $\mathbf{P}$ & $\mathrm{Ca}$ & Mg & K & Al & CTC $_{\text {Efetiva }}$ \\
\hline & $\mathrm{cm}$ & - & $-\mathrm{gk}$ & $a^{-1}$ & 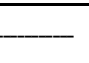 & & $\mathrm{g} \mathrm{kg}$ & & . & $\mathrm{mmol}_{\mathrm{c}}$ & & 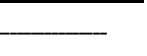 \\
\hline $\mathrm{TR}$ & $\begin{array}{r}0-20 \\
20-40\end{array}$ & $\begin{array}{l}220 \\
170\end{array}$ & $\begin{array}{l}320 \\
330\end{array}$ & $\begin{array}{l}460 \\
600\end{array}$ & $\begin{array}{r}12,1 \\
5,1\end{array}$ & $\begin{array}{l}6,2 \\
6,6\end{array}$ & $\begin{array}{l}3 \\
1\end{array}$ & $\begin{array}{l}57 \\
16\end{array}$ & $\begin{array}{r}17 \\
5\end{array}$ & $\begin{array}{l}5,0 \\
3,5\end{array}$ & $\begin{array}{l}0 \\
0\end{array}$ & $\begin{array}{l}79,0 \\
24,5\end{array}$ \\
\hline LA & $\begin{array}{r}0-20 \\
20-40\end{array}$ & $\begin{array}{l}710 \\
740\end{array}$ & $\begin{array}{l}80 \\
40\end{array}$ & $\begin{array}{l}210 \\
220\end{array}$ & $\begin{array}{l}7,7 \\
4,7\end{array}$ & $\begin{array}{l}4,8 \\
4,0\end{array}$ & $\begin{array}{l}2 \\
1\end{array}$ & $\begin{array}{l}4 \\
3\end{array}$ & $\begin{array}{l}2 \\
2\end{array}$ & $\begin{array}{l}0,5 \\
0,5\end{array}$ & $\begin{array}{l}9 \\
6\end{array}$ & $\begin{array}{l}15,5 \\
11,5\end{array}$ \\
\hline
\end{tabular}

${ }^{(1)}$ Terra Roxa estruturada eutrófica; LA - Latossolo Amarelo.

NPK. Os tratamentos testados foram NPK + micronutrientes (NPKM), NPK + Zn (NPKZn); NPK + calcário na dosagem de 1 t ha-1 (NPKC); NPK + esterco de gado na dose de 1 t ha-1 $\left(\mathrm{NPKE}_{1}\right)$; NPK +esterco de gadona dosagem de 5 t ha-1 $\left(\mathrm{NPKE}_{2}\right)$; fracionamento da adubação NPK em três aplicações ao ano (NPKF) e testemunha (NPK), deacordo com o método descrito por Gomes (1977). Os demais tratamentos foram apresentados e discutidos anteriormente por Morais (1998). A parcela experimental foi constituída de 20 plantas úteis em cada solo, utilizando-se bordaduras simples entre parcelas e blocos. As fontes e as doses de nutrientes utilizados foram a uréia ( $60 \mathrm{~kg} \mathrm{ha}^{-1}$ de $\mathrm{N}$ ), superfosfato triplo $\left(60 \mathrm{~kg} \mathrm{ha}^{-1}\right.$ de $\left.\mathrm{P}_{2} \mathrm{O}_{5}\right)$, cloreto de potássio (60 kg ha-1 de $\left.\mathrm{K}_{2} 0\right)$, sulfato de zinco (9 kg ha-1 de Zn), cl oreto de ferro ( $4 \mathrm{~kg} \mathrm{ha}^{-1}$ de Fe), tetraborato de sódio ( $2 \mathrm{~kg} \mathrm{ha}^{-1} \mathrm{de}$ ), mol ibdato de sódio ( $3 \mathrm{~kg}$ ha-1 de Mo) e sulfato de cobre ( $3 \mathrm{~kg} \mathrm{ha}^{-1} \mathrm{deCu}$ ). As doses por planta de calcário e do adubo orgânico foram, inicialmente, incorporadas à cova de plantio e, posteriormente, aplicadas a lanço, a cada três anos, no espaço compreendido entre quatro cacaueiros. Os demais tratamentos, com exceção de micronutrientes, foram aplicados a lanço, anualmente, no mesmo local. A adubação com micronutrientes foi realizada em intervalos de cinco anos, desde a implantação das lavouras de cacau.

Foram avaliados o número total de frutos produzidos (Tfp), a perda de frutos atacados pela enfermidade vassoura-de-bruxa (VB) e a produção anual de amêndoas secas de cacau (Prod) do período de 1988 a 1993. A análise de variância foi efetuada, utilizando o model o de grupos de experimentos, sendo as correlações entre essas variáveis e os fatores ano, solo e tratamentos (Trat) também determinadas. A porcentagem de frutos infectados com a vassoura-debruxa (VB\%) foi calculada a partir da fórmula:

$$
\text { VB } \%=F i \times 100 / T f p \text {, }
$$

em que:

$\mathrm{FI}=$ Número de frutos perdidos por infecção com vassoura-de-bruxa; $\mathrm{Tfp}=$ Número total de frutos produzidos.

\section{RESULTADOS E DISCUSSÃO}

O quadro 2 mostra o efeito dos tratamentos sobre os componentes de produção e sanidade do cacaueiro. Não houve interaçãosignificativa entre os parâmetros anal isados eo solo ou ano de condução do experimento. Os mel hores tratamentos foram o fracionamento da adubação mineral e a aplicação de zinco, com incrementos $(P<0,05)$ na produtividadedeamêndoas secas da ordem de $27,5 \%$ e $10,9 \%$, respectivamente, na média do período de 1988 a 1993. O efeito dos tratamentos no número total de frutos produzidos apresentou tendência semelhante. Não foram verificados benefícios da calagem ou da adubação orgânica sobre a produção de amêndoas secas, contrariando os resultados obtidos durante a fase de crescimento do cultivo (Morais, 1988). Morais \& Pereira (1986) mostraram que o fracionamento da adubação NPK diminui a perda de nutrientes por lixiviação, aumentando, em conseqüência, a absorção pel o cacaueiro, conforme indicado por diferenças entre tratamentos no teor de nutrientes em folhas selecionadas. Campos (1982), em experimento de casa de vegetação, demonstrou a ocorrência de deficiência de Zn em plântulas de cacau cultivadas em solos de baixa fertilidade da Amazônia. Por outro lado, a experiência adquirida na região tem revelado que mudas de cacau transplantadas para áreas recémquei madas apresentam deficiências local izadas de Zn, sendo este efeito de curta duração em virtude da solubilidade das cinzas adicionadas ao solo. Segundo Morais et al. (1978), o excesso de calagem provoca deficiências de micronutrientes, especialmente $Z n$ e $\mathrm{Fe}$, em plântulas de cacau cultivadas em casa de vegetação, devido à el evação do pH acima de 7,0. No processo de queima da floresta, a incorporação de cinzas é desuniforme, principalmente pelo emprego da prática de encoi varamento de resíduos vegetais, o que também contribui para el evar o pH do solo para valores superiores a 7,0 em determinados locais da área.

As aplicações de uma mistura de micronutrientes e de esterco de gado na dose de $5 \mathrm{t}$ ha-1 diminuíram $(P>0,05)$ a porcentagem de infecção de frutos atacados pela vassoura-de-bruxa, tendo ocorrido os 
Quadro 2. Número de frutos produzidos (Tfp), porcentagem de frutos atacados pela doença vassoura-debruxa (VB) e produção de amêndoas secas de cacau, considerando os tratamentos. Média de 1988 a 1993(1)

\begin{tabular}{lcccc}
\hline Tratamento & Produção de amêndoas & Tfp & VB & índice de produção \\
\hline & $\mathrm{kg} \mathrm{ha}^{-1}$ & Frutos/parcela & \\
NPKF & $864,6 \mathrm{a}$ & $559 \mathrm{a}$ & $13,4 \mathrm{ab}$ & 127,5 \\
NPKZn & $752,2 \mathrm{~b}$ & $539 \mathrm{ab}$ & $13,2 \mathrm{ab}$ & 110,9 \\
NPKE & $737,1 \mathrm{bc}$ & $531 \mathrm{ab}$ & $14,0 \mathrm{a}$ & 108,7 \\
NPKM & $731,7 \mathrm{bc}$ & $477 \mathrm{bc}$ & $11,7 \mathrm{bc}$ & 107,9 \\
NPKC & $727,4 \mathrm{bc}$ & $501 \mathrm{abc}$ & $14,5 \mathrm{a}$ & 107,2 \\
NPKE & $709,8 \mathrm{bc}$ & $473 \mathrm{bc}$ & $11,1 \mathrm{c}$ & 104,6 \\
NPK (Test.) & $678,3 \mathrm{c}$ & $418 \mathrm{c}$ & $13,6 \mathrm{a}$ & 100,0 \\
C.V. (\%) & 25,7 & 25,6 & 35,4 & - \\
\hline
\end{tabular}

(1) NPKF = adubação NPK fracionada em três aplicações ao ano; NPKZn = adubação NPK + Zinco; NPKE ${ }_{1}=$ adubação NPK + esterco de gado na dose de 1 t ha ${ }^{-1}$; NPKM = adubação NPK + micronutrientes; NPKC = adubação NPK + calcário na dose de 1 t ha-1; NPKE $_{2}$ $=$ adubação NPK + esterco de gado na dose de $5 \mathrm{t}$ ha ${ }^{-1}$. Letras diferentes na mesma coluna indicam diferenças significativas ao nível de $5 \%$ pelo teste de Duncan.

índices mais elevados de perda de frutos devidos a essa doença (Quadro 2) nos tratamentos com cal cário, com adubo orgânico na dosagem de 1 t ha-1 e na testemunha. Em trabal ho anterior, Morais (1998) demonstrou o efeito do N, do P e do K no índice de infecção de frutos do cacaueiro; resultados que mostram que o efeito da adubação NPK pode ser mel horado pela aplicação adicional demicronutrientes ou de matéria orgânica em al tas dosagens. A literatura descreve os benefícios da aplicação foliar de $\mathrm{Cu}$ em áreas de alto potencial de inoculação por Phytophtora palmivora e Crinipellis perniciosa, agentes causais das enfermidades podridão parda evassoura-de-bruxa do cacaueiro, respectivamente, o que parcialmente explica os resultados significativos obtidos pelo tratamento com micronutrientes sobre a infecção de vassoura-de-bruxa (Bastos \& Evans, 1979; Figueiredo \& Lellis, 1980; Lellis et al., 1983; L awrenceet al., 1991).

O quadro 3 contém os coeficientes de correlação

Quadro 3. Coeficiente de correlação ( $r$ ) entre produção de amêndoas secas de cacau (Prod), total de frutos produzidos (Tfp), porcentagem de infecção de frutos por vassoura-de-bruxa (VB\%), ano e tratamentos fertilizantes (Trat.)

\begin{tabular}{lccc}
\hline Variável & Prod & Tfp & VB \% \\
\hline Prod & $1,00^{* *}$ & $0,77^{*}$ & $-0,48$ \\
Tfp & $0,77^{*}$ & $1,00^{* *}$ & $-0,06$ \\
VB & $-0,48$ & $-0,06$ & $1,00^{* *}$ \\
Trat & 0,03 & 0,11 & $-0,05$ \\
Ano & $-0,29$ & 0,07 & $0,81^{* *}$ \\
\hline
\end{tabular}

(r) entre as variáveis dependentes, Tfp, VB\%, Prod, e independentes, Ano e Trat. Deve-se observar a existência de correlação significativa entreTfp eProd $\left(r=0,77^{*}\right)$, apesar de a enfermidade vassoura-debruxa diminuir o número de frutos disponíveis para beneficiamento e comercial ização. O maior coeficiente de correlação foi encontrado entre as variáveis VB e Ano $\left(r=0,81^{* *}\right)$, devido à intensificação da enfermidade com a idade do cacaueiro, especial mente após o quinto ano de campo.

\section{CONCLUSÕES}

1. O fracionamento da adubação NPK ea aplicação de $\mathrm{Zn}$ incrementaram a produtividade do cacaueiro em sol os da Amazônia;

2. A adubação orgânica, na dosagem de $5 \mathrm{t} \mathrm{ha-1,} \mathrm{e}$ uma mistura de micronutrientes contribuíram para diminuir oíndice de frutos atacados pela enfermidade vassoura-de-bruxa; a adubação orgânica, na dose de 1 t ha-1, e a aplicação de cal cário não alteraram esse índice;

3. Correlações positivas foram obtidas entre ototal defrutos produzidos e a produção anual de amêndoas secas de cacau e entre porcentagem de frutos atacados pela vassoura-de-bruxa e ano de condução do experimento.

\section{LITE RATURA CITADA}

BASTOS, C.N. \& EVANS, H.C. Resultados preliminares sobre o estabelecimento de um sistema de controle da vassoura-debruxa na Amazônia. Belém, CE PLAC, 1979. 12p. (Comunicado Técnico, 12) 
CAMPOS, A.X. Avaliação da fertilidade de solos cacaueiros na Amazônia. Belém, CEPLAC, 1982. 17p. (ComunicadoTécnico, 22)

FALESI, I,C. Solos da rodovia transamazônica. Belém, IPEAN, 1972. 19p. (Boletim Técnico 55)

FIGUEIREDO, J.M. \& LELLIS, W.T. Controle da podridão parda do cacaueiro com fungicidas cúpricos. R. Theobroma, 10:117121, 1980.

GARCIA, J J.; MORAIS, F.I.O.; ALMEIDA, L.C. \& DIAS, J.C. Sistema de produção do cacaueiro na Amazônia Brasileira. Belém, CEPLAC, 1985. 118p.

GOMES, F.P. Curso de estatística experimental. São Paulo, Nobel, 1977. 430p.

LAWRENCE, J.S.; CAMPELO, A.M.F.L. \& FIGUEIREDO, J.M. Enfermidades do cacaueiro. II- Doenças fúngicas que ocorrem nas fol has, ramos e tronco. Agrotrópica, 3:1-14, 1991.

LELLIS, W.T.; FI GUEIREDO, J.M. \& PEREIRA, J .L.M. Progressos no controle da podridão parda na Bahia, Brasil. R. Theobroma, 13:105-111, 1983.
MORAIS, F.I O. Efeito de fertilizantes e corretivos no crescimento e produção do cacaueiro em solos da Amazônia Brasileira. In: CONFERENCIA INTERNACIONAL DE PESQUISAS EM CACAU, 10., Santo Domingo, República Dominicana, 1987. Proceadings. England, Cocoa Producers'Alliance, 1988. p.247-251.

MORAIS, F.I.O. \& PEREIRA, G.C. Respostas do cacaueiro à aplicação de fertilizantes e corretivos nas condições da Amazônia brasileira. I. Crescimento e produção inicial. R. Theobroma, 16:65-73, 1986.

MORAIS, F.I.O., SANTANA, C.L. \& CHEPOTE, R.E. Efeitos da aplicação de calcário e fósforo no crescimento do cacaueiro em condições de casa de vegetação. R. Theobroma, 8:73-75, 1978.

MORAIS, F.I O. Respostas do cacaueiro à aplicação de N, P eK em dois solos da Amazônia Brasileira. R. Bras. Ci. Solo, 22:6369. 1998.

NEVES, A.D. \& BARBOSA, R.C.M. Solos dos pólos cacaueiros da Amazônia. Belém, CE PLAC, 1982. 13p. (ComunicadoTécnico Especial, 4)

VIEIRA, L.S., SANTOS, W.H.P., FALESI, I.C. \& FILHO, J.P.S.O. Levantamento de reconhecimento dos solos da região Bragantina, Estado do Pará. Pesq. agrop. bras., 2:1-63, 1967. 